\title{
Potenzial und Grenzen \\ von Zuwanderung \\ in die Pflege
}

Stefan Sell

6.1 Von (un)sicheren Bedarfen - 86

6.2 Von solchen und anderen „Ausländern“ sowie den rechtlichen Rahmenbedingungen der Zuwanderung - 87

6.3 Ausländische Pflegekräfte sind schon da, aber es ist überschaubar - 89

6.4 Die osteuropäische Pendelmigration als "Schattensäule“ der Zuwanderung in der Altenpflege bzw. häuslichen Betreuung - 92

6.5 Die Rekrutierung ausländischer Pflegekräfte oder: Vom Entstehen einer eigenen Branche; viele Klimmzüge, überschaubare Resultate - 93

Literatur - 99 


\section{- Zusammenfassung}

Bereits heute sind wir mit einem eklatanten Mangel an Pflegefachkräften in der Altenpflege konfrontiert. Und gerade in diesem Bereich wird es aufgrund der demografischen Entwicklung im Zusammenspiel mit Verschiebungen der Pflegearrangements eine erhebliche Zunahme des Bedarfs an Pflegekräften geben. Vor diesem Hintergrund werden viele Hoffnungen mit einer Ausweitung der Zuwanderung von Pflegekräften aus dem Ausland verbunden. Die nimmt in den vergangenen Jahren durchaus $z u$ - aber quantitativ ist sie nur ein überschaubarer und zugleich fragiler Baustein der notwendigen Personaldeckung. Aus dem pflegerischen Alltag werden zugleich zahlreiche Spannungen und Konflikte berichtet. Insgesamt wird man intensive Bemühungen einer Rekrutierung ausländischen Pflegepersonals betreiben müssen, aber man sollte sich der strukturellen Grenzen bewusst sein - vor allem der Sprachbarrieren und der kulturellen Probleme im Arbeitsalltag. Eine einseitige Absenkung der Anforderungen an Pflegekräfte aus dem Ausland liegt zwar nahe, sollte aber unbedingt vermieden werden.

At present we are confronted with a blatant shortage of skilled staff in nursing care for the elderly. And due to demographic developments in conjunction with shifts in nursing arrangements, there will be a considerable increase in the demand for nursing staff. Against this background, many hopes are associated with an increase in the immigration of nursing staff from abroad. However, while there is indeed a certain increase in this area, in quantitative terms it is a rather small and at the same time fragile component of the necessary personnel coverage. Moreover, numerous tensions and conflicts have been reported from everyday nursing care. Overall, intensive efforts will have to be made to recruit foreign nursing staff, but one should be aware of the structural limits. These concern above all language barriers and cultural problems in everyday working life. A reduction in the demands placed on nursing staff from abroad is obvious, but should be avoided at all costs.

\subsection{Von (un)sicheren Bedarfen}

Es ist immer wieder beeindruckend, wie in der öffentlichen Diskussion Zahlen über den Personalbedarf in „der" Altenpflege vorgetragen werden, die eine völlig haltlose Genauigkeit vortäuschen, aber die Sehnsucht gerade der Medien nach möglichst der einen Zahl zu befriedigen versuchen. Wie viele Pflegekräfte fehlen heute und wie viele werden wir in zehn oder zwanzig Jahren brauchen? Und immer wieder versuchen Wissenschaftler, diese Nachfrage zu bedienen. Nur ein Beispiel:

Mit Blick auf den Fachkräftebedarf in der ambulanten und stationären Altenpflege ${ }^{1}$ sind Flake et al. (2018) zu diesem Ergebnis gekommen: „Im Basisszenario erhöht sich der Bedarf an Fachkräften von 2015 bis 2035 für die pflegerische Versorgung um gut 150.000 auf insgesamt knapp 494.000 Personen ... In einem Szenario, in dem sich der Gesundheitszustand positiv mit der Lebenserwartung entwickelt, steigt er immerhin um rund 130.000 auf insgesamt knapp 473.000 Personen an." (Flake et al. 2018, S. 34). Das hört sich doch sehr exakt an.

Aber man findet in der Studie dann auch diesen wichtigen Hinweis: „Der allein durch den demografischen Wandel getriebene Bedarf an Fachkräften für die Pflege steigt je nach Szenario somit um $44 \%$ oder $38 \%$, selbst wenn unterstellt wird, dass der heutige Personalschlüssel konstant bleibt." (Flake et al. 2018, S. 34). Der entscheidende Punkt lautet: selbst wenn der heutige Personalschlüssel konstant bleibt. Genau darüber wird aber zu Recht intensiv diskutiert. Hinzu kommen Fragen, wie das Mischungsverhältnis zwischen den unterschiedlichen Pflegearrangements in der $\mathrm{Zu}$ kunft aussehen wird; ob, und wenn ja, welche Verschiebungen es geben wird. Und wie sieht es mit den osteuropäischen Betreuungskräften aus, die heute in vielen Privathaushalten unterhalb des offiziellen Radars unterwegs sind? Vor

Man muss bei den Zahlen berücksichtigen, dass das Institut der deutschen Wirtschaft hier "nur" den Bedarf an Pflegefachkräften berechnet hat, die Pflegehilfskräfte sind ausgeklammert. 
diesem hier nur anzudeutenden hyperkomplexen Hintergrund sollte man allen Vorhersagen mit größter Skepsis begegnen, vor allem, wenn sie Punktschätzungen in die Welt werfen. Vgl. zu der ganzen Thematik ausführlicher die Ausarbeitung „Wie viele Pflegekräfte in der Altenpflege müssen es denn sein? Von (un)sicheren Bedarfen und beweglichen Zielen bei der Diskussion über den bestehenden und kommenden Mangel an Pflegepersonal“" (Sell 2019).

\subsection{Von solchen und anderen "Ausländern" sowie den rechtlichen Rahmenbedingungen der Zuwanderung}

In der pflegepolitischen Diskussion wird immer wieder die Forderung vorgetragen, dass mehr „ausländische“ Pflegekräfte nach Deutschland kommen müssen, um den heute schon bestehenden und vor allem den zusätzlich zu erwartenden Personalbedarf auch tatsächlich mit Pflegekräften decken zu können, da die einheimischen Ressourcen dafür nicht ausreichen (würden). Aber der Begriff „Ausländer“ hört sich homogener an als er in der Praxis und auch in der Statistik ${ }^{2}$ dann ist und es gibt mit Blick auf die Möglichkeit, Pflegekräfte „aus dem Ausland“ nach Deutschland zu holen, ganz unterschiedliche "Ausländer“, mit entsprechend divergierenden Folgen für die Beschäftigung dieser Menschen in Deutschland.

Wenn es wie auch in diesem Beitrag um eine gezielte Anwerbung von Pflegekräften aus dem Ausland geht, dann kommen so-

2 Das wird im $>$ Abschn. 6.3 deutlich bei der Analyse der „ausländischen Pflegekräfte" auf der Basis der offiziellen Statistiken. Hier wird jeder, der eine ausländische Staatsangehörigkeit hat, in einer Kategorie zusammengefasst, die nicht danach unterscheidet, ob es sich um jemanden handelt, der als Pflegekraft zugewandert ist oder der gleichsam als "Inländer" hier aufgewachsen ist, aber nicht die deutsche Staatsangehörigkeit hat. wohl Personen aus den EU-Mitgliedsstaaten wie auch aus Staaten außerhalb der EU in Frage. $^{3}$ Hier muss man dann unterscheiden hinsichtlich aufenthalts- und arbeitserlaubnisrechtlicher Fragen sowie der Frage einer Berufsanerkennung. ${ }^{4}$

Ein Blick zurück ist hilfreich, auch um die Veränderungen einschätzen zu können, die mittlerweile bereits stattgefunden haben. In der Zeit vor der Beschäftigungsverordnung vom 1. Juli 2013 war es in Deutschland nur in Ausnahmefällen gestattet, ausländisches Pflegepersonal in Pflegeeinrichtungen anzustellen. Gemäß den beschränkenden $\mathrm{Zu}-$ wanderungsregelungen, die bis zum Inkrafttreten galten, durften Pflegekräfte aus NichtEU-Staaten lediglich auf Grundlage gesonderter Vermittlungsabsprachen in Deutschland arbeiten. Doch auch Pflegekräfte aus den osteuropäischen EU-Beitrittsländern mussten einige Hürden meistern, ehe sie als "vollwertige“ Pflegerinnen und Pfleger galten. Mittlerweile gilt für alle EU-Mitgliedsstaaten die Arbeitnehmerfreizügigkeit, sodass für Menschen aus diesen Ländern keine Arbeitserlaubnis mehr erforderlich ist. Sie sind inländischen Arbeitnehmern gleichgestellt. ${ }^{5}$

Bleibt die Gruppe der Pflegekräfte, die aus Nicht-EU-Staaten angeworben werden sollen. Hier sind (bislang) die Hürden generell für Arbeitgeber teilweise sehr hoch bis unüberwindlich. Die Pflegebranche befindet sich aber in der privilegierten Position, auch von außerhalb der Europäischen Union Pflegefachkräfte anwerben zu können, da sowohl die qualifizierten Tätigkeiten in der Gesundheits- und Krankenpflege als auch die Altenpflegefachkräfte auf der Liste der Mangelberufe stehen, in die seit der Reform der Beschäftigungsverordnung

Eine dritte Gruppe wären Personen mit Migrationshintergrund, die bereits in Deutschland leben, entweder mit einem bereits geklärten aufenthaltsrechtlichen Status, beispielsweise anerkannte Asylbewerber oder Arbeitsmigranten oder deren Kinder.

4 Vgl. dazu auch Deutscher Paritätischer Wohlfahrtsverband (2018).

5 Dies gilt auch für Staatsangehörige aus Island, Norwegen, Schweiz und Liechtenstein. 
von 2013 eine Zuwanderung nach Deutschland mit Zustimmung der Zentralen Auslands- und Fachvermittlung ohne Vorrangprüfung erfolgen kann (Bonin et al. 2015, S. 63).

Pflegefachkräfte aus ausgewählten NichtEU-Staaten können auf der Basis bilateraler Vermittlungsabsprachen der Bundesregierung angeworben werden. Umgesetzt wird dies von der Bundesagentur für Arbeit. Damit muss beachtet werden, dass die Anwerbung und Arbeitsvermittlung in bzw. aus 57 Ländern, darunter viele afrikanische, arabische, süd- und mittelamerikanische Staaten, für eine Beschäftigung in Gesundheits- und Pflegeberufen in Deutschland nur von der Bundesagentur für Arbeit durchgeführt werden darf. ${ }^{6}$ Außerdem muss man darauf hinweisen, dass eine weltweite Suche nach möglichen Pflegekräften für Deutschland begrenzt wird (bzw. werden soll) durch den „WHO Code of Practice“, über den definiert wird, in welchen Ländern kein bedenklicher Engpass von Gesundheitspersonal vorliegt - denn nur die kommen (eigentlich) als Anwerbeländer in Frage. ${ }^{7}$

Aber selbst wenn man die Rekrutierung von ausländischen Pflegekräften organisiert bekommt, bleibt als nächste rechtliche Hürde die Frage der Anerkennung der beruflichen Qualifikation. Die rechtliche Grundlage dafür ist das „Gesetz über die Feststellung

6 Die derzeit 57 Länder, für die diese Regelung gilt, sind aufgelistet in der Anlage zu § 38 BeschV, www. gesetze-im-internet.de/beschv_2013/anlage_1. html.

7 Managing health workforce migration - The Global Code of Practice: www.who.int/hrh/migration/ code/practice/en/. Die Regeln, die einen Brain-Drain aus den Ländern verhindern sollen, die selbst einen Mangel haben, müssen letztendlich freiwillig umgesetzt werden. Die bisherigen Erfahrungen damit sind mehr als ernüchternd, so beispielsweise die Evaluierungsergebnisse von Siyam et al. (2013). Vgl. auch die Ergebnisse von Tankwanchi et al. (2014). In Deutschland ist es so, dass eine Anwerbung aus diesen Ländern nicht grundsätzlich „verboten“ ist, § 38 BeschV regelt in Verbindung mit der Anlage zu diesem Paragrafen nur, dass eine Anwerbung über die Bundesagentur für Arbeit laufen muss (und damit kann). der Gleichwertigkeit von Berufsqualifikationen “ $(\mathrm{BQFG}){ }^{8}$

Fachkräfte im Bereich Pflege gehören zu den reglementierten Berufen. Bei den Pflegeberufen ist die Feststellung der Gleichwertigkeit zwingende Voraussetzung dafür, dass der Beruf in Deutschland ausgeübt werden darf (entsprechend auch die ausländerrechtliche Regelung in $\$ 6$ Abs. 2 BeschV). Ausreichende Sprachkenntnisse gehören zu den Voraussetzungen für die Anerkennung als Fachkraft ( $\$ 2$ Abs. 1 Nr. 4 AltPflG $)^{9}$. Das bedeutet in der Regel Niveau B2. Die Frage der Berufsanerkennung ist auch für die Beschäftigung von „EU-Ausländern“ relevant.

Im Grunde gibt es zwei Wege: die Berufsausbildungsanerkennung oder die Verpflichtung zu einer erneuten Berufsausbildung in Deutschland. Auf ein Anerkennungsverfahren haben nur diejenigen eine Chance, die bereits eine anerkannte und abgeschlossene berufliche Ausbildung im jeweiligen Staat vorweisen können. ${ }^{10}$ Abschlüsse aus Drittstaaten unterliegen stets einer Einzelfallprüfung. Dabei wird geprüft, ob der ausländische gegenüber dem deutschen Abschluss wesentliche Unterschiede aufweist. Ist dies der Fall, werden vorhandene Berufserfahrungen einbezogen. Es ist möglich, zwischen einem sogenannten Anpassungslehrgang und einer Kenntnisprüfung zu wählen, um die Anerkennung zu bekommen. „Um ... bei der Kenntnisprüfung antreten zu können und diese abzulegen, bedarf es eines Vorberei-

8 Verordnung über die Beschäftigung von Ausländerinnen und Ausländern: www.gesetze-im-internet. $\mathrm{de} / \mathrm{bqfg} /$.

9 Gesetz über die Berufe in der Altenpflege (Altenpflegegesetz - AltPflG): https://www.gesetze-iminternet.de/altpflg/BJNR151310000.html.

10 Auch hier gibt es einen Unterschied zwischen EUund Nicht-EU-Staaten. Kommt die Arbeitskraft aus einem Nicht-EU-Staat, dann muss der Unterschied zwischen dem Ausbildungsgang in Deutschland und denen des Herkunftslandes näher geprüft werden. Außerdem: Sollte der Nachweis einer praktischen Berufsausübung nicht möglich sein, dann wird die absolvierte Pflegeausbildung eventuell nur zu gewissen Teilen anerkannt und es wird nur eine Teilgleichwertigkeit ausgesprochen. 
tungskurses, der sich erfahrungsgemäß auf ca. ein Jahr beläuft. Der schnellste Weg zu einer vollen Gleichwertigkeit der Ausbildungsgänge führt über die Kenntnisprüfung. Hierbei sind die ausländischen Pflegekräfte aber enormem Druck und hohem Risiko des Nichtbestehens ausgesetzt“, so Nürnberg und Traoré (2019, S. 125).

Die Berufsanerkennung in Deutschland erfolgt als Gesundheits- und Krankenpfleger/ -in. Hierzu ist ein Anerkennungsverfahren in der Gesundheits- und Krankenpflege wie auch bei einer Tätigkeit in der Altenpflege möglich. Denn: Das Berufsbild des examinierten Altenpflegers existiert im internationalen Ausbildungssystem nicht.

\subsection{Ausländische Pflegekräfte sind schon da, aber es ist überschaubar}

„Wir werden bis zu 50.000 zusätzliche Pflegekräfte brauchen. Da werden wir auch im Ausland suchen müssen“, so wird Bundesgesundheitsminister Jens Spahn (CDU) zitiert. Es sei kaum mehr möglich, in Deutschland ein Krankenhaus oder eine Pflegeeinrichtung ohne ausländische Pflegekräfte zu betreiben. Besonders im Kosovo und in Albanien gibt es laut Spahn ein hohes Potenzial an jungen Fachkräften: „Dort ist die Pflegeausbildung häufig besser als wir denken."11

Vor diesem Hintergrund lohnt ein Blick auf die Zahlen. Die findet man beispielsweise in dieser Antwort der Bundesregierung auf eine Kleine Anfrage im Bundestag: „Ausländische Pflegekräfte in Deutschland“" (BundestagsDrucksache 19/2455 vom 04.06.2018). Dort wird berichtet:

„Nach Angaben der Statistik der BA waren im Juni 2017 bundesweit rund 128.000 ausländische Pflegekräfte sozialversicherungspflichtig und 5.900 ausschließlich geringfügig beschäftigt." Im Juni 2013 waren es 74.046 sozialversi-

11 Vgl. zu den Ausführungen des Ministers den Beitrag von Sell (2018a). cherungspflichtig und 5.366 ausschließlich geringfügig beschäftigte ausländische Pflegekräfte.

Von den 128.000 ausländischen Pflegekräften im Juni 2017 kamen 18.334 aus den vom Minister angesprochenen Balkanstaaten, also Albanien, Bosnien und Herzegowina, Kosovo, Mazedonien und Serbien (•Tab. 6.1).

Zur Bewertung dieser Zahlen sollten wir uns verdeutlichen, dass es sich bei der Zahl 128.000 ausländische Pflegekräfte (Stand: Juni 2017) um Beschäftigte in der Krankenhausund Altenpflege zusammen handelt, also in einem Bereich, wo wir derzeit 1,7 Mio. Beschäftigte insgesamt verzeichnen. Dies relativiert dann die Bedeutung dieser Zahl erheblich.

Für den Juni 2018 werden von der Bundesagentur für Arbeit insgesamt knapp 143.500 sozialversicherungspflichtig beschäftigte Pflegekräfte mit ausländischer Staatsangehörigkeit ausgewiesen. $^{12}$ Darunter mehr als 70.800, die in der Altenpflege beschäftigt waren (- Abb. 6.1). Aber auch hier gilt die bereits getroffene Feststellung: In der Beschäftigungsstatistik werden alle in der Pflege (sozialversicherungspflichtig) beschäftigten Personen als „Ausländer“ ausgewiesen, wenn sie eine ausländische Staatsangehörigkeit haben.

Hilfreich an dieser Stelle ist ein Blick zurück in die Fachdiskussion über Chancen und Grenzen einer Zuwanderung in der Pflege. Dazu die Analyse „Können Pflegekräfte aus dem Ausland den wachsenden Pflegebedarf decken? Analysen zur Arbeitsmigration in Pflegeberufen im Jahr 2010“ (Afentakis und Maier 2014). Ein höchst relevanter Befund: „In den letzten Jahren war die Zuwanderung von Arbeitsmigrantinnen/-migranten in Pflegeberufen ... kontinuierlich rückläufig: Während zwischen 1988 und 1995 jährlich durchschnittlich 6.000 Arbeitsmigrantinnen/-migranten nach Deutschland kamen, ging die Zuwanderung zwischen 1996 und 2004 auf durchschnittlich 4.000 je Jahr zurück. Zwischen 2005 und 2009 sind jährlich durchschnittlich nur noch 2.000 Arbeitsmigrantinnen/-migranten nach

12 Vgl. Bundesagentur für Arbeit (2019). 


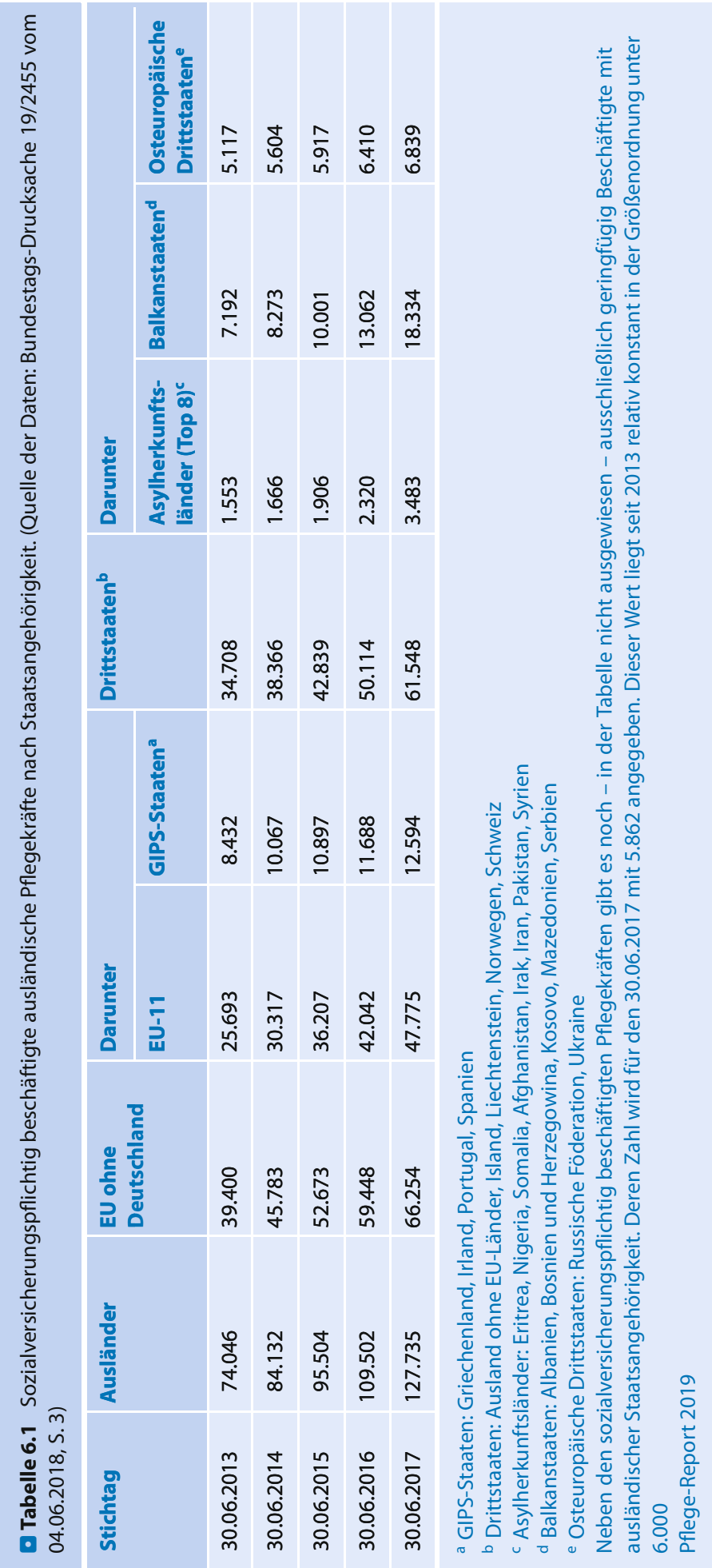




\section{Sozialversicherungspflichtig Beschäftigte in der Gesundheits- und Krankenpflege insgesamt und darunter Ausländer}

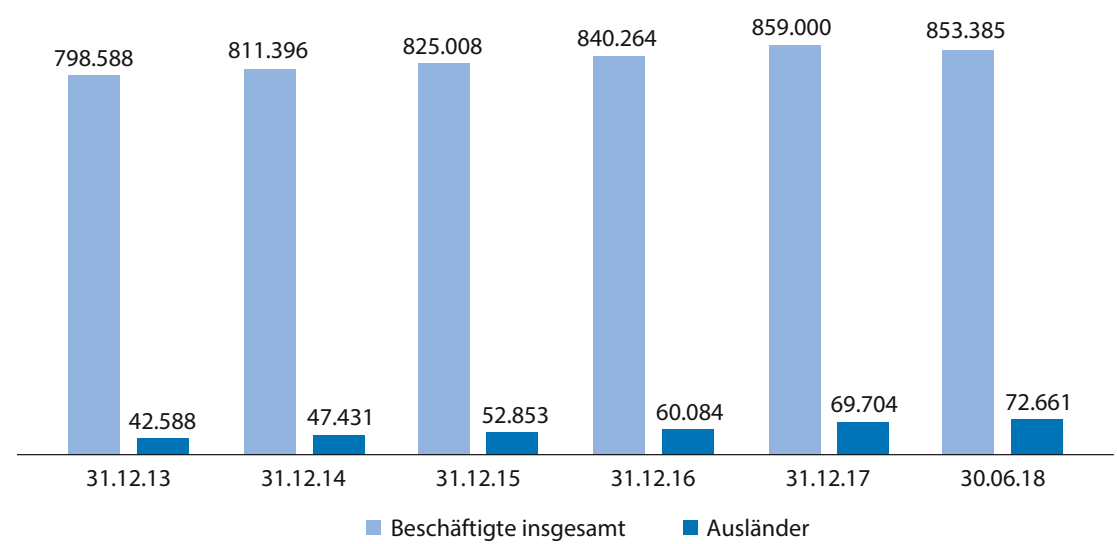

Sozialversicherungspflichtig Beschäftigte in der Altenpflege insgesamt und darunter Ausländer

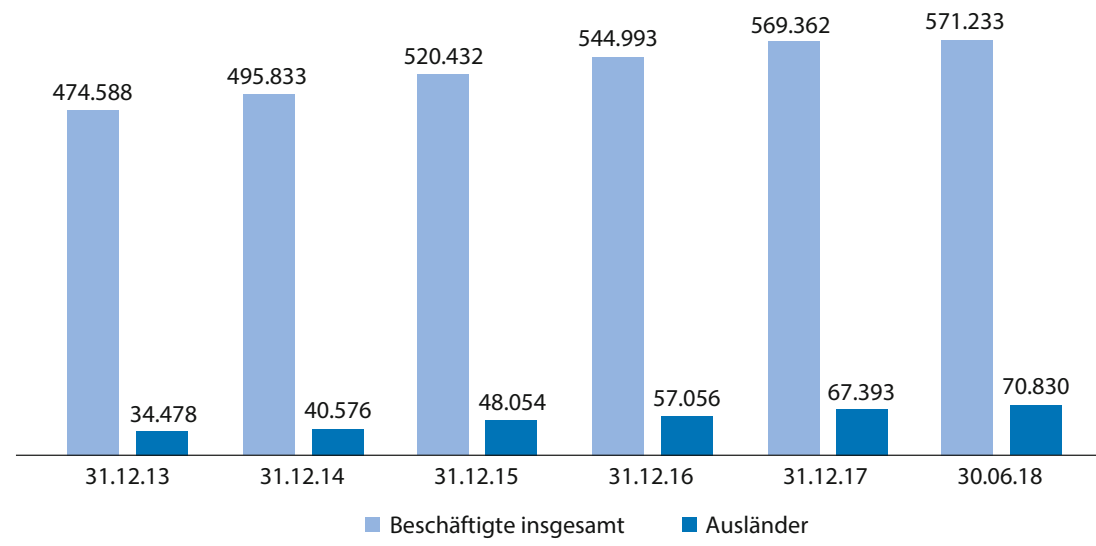

Quelle der Daten: Bundesagentur für Arbeit: Beschäftigte nach Berufen

- Abb. 6.1 Sozialversicherungspflichtig Beschäftigte in der Gesundheits- und Krankenpflege insgesamt und darunter Ausländer

Deutschland zugewandert. Auch von den mindestens einjährig ausgebildeten Pflegekräften, die einen Pflegeberuf ausübten (Stayer), kamen die meisten in den 1990er-Jahren nach Deutschland (52,3\%)“ (Afentakis und Maier 2014, S. 178). Im Fazit wird ausgeführt:
Die qualifizierte Zuwanderung in den Pflegeberufen konzentrierte sich auf die Berufsordnungen der Gesundheits- und Krankenpflegerinnen/-pfleger - die Altenpflege ist hier nur gering vertreten. Für die Vergangenheit gilt: Arbeitsmigrantinnen/-migranten aus der ehe- 
maligen Sowjetunion und den östlichen EUStaaten stellten den Großteil der in den Pflegeberufen beschäftigten Arbeitsmigrantinnen/ -migranten. In den letzten zehn Jahren vor 2010 profitierten die Pflegeberufe zunehmend weniger von einer Arbeitsmigration.

„Wenn sich diese Entwicklung fortsetzt, könnte die Arbeitsmigration in Zukunft nur zu einem geringen Teil dazu beitragen, die Personalengpässe in den Pflegeberufen zu beseitigen. Die Analysen zur beruflichen Flexibilität verdeutlichen vielmehr, dass es langfristig aus gesamtwirtschaftlicher Perspektive lohnenswerter ist, Pflegekräfte in Deutschland auszubilden, da sie anschließend auch überwiegend einen Pflegeberuf ausüben, sofern sie erwerbstätig sind (zu 82,9\%). Dies gilt insbesondere für Pflegekräfte mit einer zwei- beziehungsweise dreijährigen Ausbildung. Dies setzt bei einer immer geringer werdenden Zahl an Jugendlichen aber auch voraus, dass die Pflegeberufe im Vergleich zu anderen möglichen Berufen an Attraktivität gewinnen“ (Afentakis und Maier 2014, S. 179).

Für den aktuellen Stand kann man hingegen gemessen an der sozialversicherungspflichtigen Beschäftigung von ausländischen Pflegekräften deutlich höhere Zahlen feststellen:

2014: +10.086, 2015: +11.372, 2016: +13.998 und 2017: $+18.233^{13}$.

Allerdings muss auch hier beachtet werden, dass die Altenpflege unterdurchschnittlich vertreten ist, die Beschäftigung konzentriert sich auf den Krankenhausbereich.

\subsection{Die osteuropäische Pendelmigration als „Schattensäule“ der Zuwanderung in der Altenpflege bzw. häuslichen Betreuung}

Wenn wir über Zuwanderung in der Pflege sprechen, kommen wir um den Schattenbe-

13 Vgl. Bundesagentur für Arbeit (2019). reich der aus osteuropäischen Ländern stammenden Pflege- und Betreuungskräfte, die in den Haushalten pflegebedürftiger Menschen leben und deren Arbeit unter dem Schlagwort „24 Stunden-Pflege" immer wieder thematisiert wird, nicht herum. Es handelt sich um eine gewaltige Entlastung für das bestehende Pflegesystem, denn deren Arbeit stabilisiert die bestehende Verteilungsstruktur, dass weit über $70 \%$ der Pflegebedürftigen zu Hause versorgt werden. Entweder von den pflegenden Angehörigen allein (aber zuweilen eben mit Rückgriff auf osteuropäische Betreuungskräfte) oder unter partieller Zuhilfenahme ambulanter Pflegedienste.

Nun sollte man nicht vorschnell annehmen, dass wir überhaupt eine valide Zahl haben, um wie viele Menschen es sich handelt. In der Antwort auf eine Anfrage im Deutschen Bundestag „Arbeitsbedingungen von im Haushalt lebenden Pflegekräften“" (BundestagsDrucksache 19/6792 vom 02.01.2019) heißt es seitens der Bundesregierung auf die Fragen „Wie viele sogenannte Live-in-Pflegekräfte gibt es nach Kenntnis der Bundesregierung in Deutschland und wie hoch war die Anzahl vor fünf, zehn, 15 und 20 Jahren?“ und „Wie viele ,24-Stunden-Pflegekräfte ' arbeiten nach Kenntnis der Bundesregierung im Jahr als Selbstständige, und wie viele sind angestellt?" sowie "Wie viele Agenturen zur Anwerbung von im Haushalt lebenden Pflegekräften sind der Bundesregierung in Deutschland bekannt" lapidar: „Der Bundesregierung liegen hierzu keine Erkenntnisse vor."

Das ist nun schon mehr als erstaunlich, wenn man sich die in der Literatur immer wieder genannten Zahlen anschaut: Nach Schätzungen beschäftigen mehr als 200.000 Haushalte in Deutschland eine Betreuungskraft aus Osteuropa - legal oder illegal. Entstanden sei eine riesige rechtliche Grauzone, so das German-Polish Centre for Public Law and Environmental Network (GP PLEN) 2018. Der Studie von Hielscher et al. (2017) kann man diese Größenordnung entnehmen: In schätzungsweise 163.000 Privathaushalten lebt eine osteuropäische Hilfskraft für eine „Rund-um- 
die-Uhr“-Betreuung eines Pflegebedürftigen. „Schätzungen gehen von ungefähr $150.000 \mathrm{mi}-$ grantischen Haushaltshilfen in deutschen Familien aus, die wenigsten davon in regulär geregelten Arbeitsverhältnissen“, so Malsburg und Isfort (2014). ${ }^{14}$

Und man muss bei diesen Zahlen berücksichtigen, dass es sich um die Haushalte handelt, in denen man eine entsprechende Beschäftigung vermutet. Die betroffenen Betreuungskräfte bleiben nun aber immer nur eine Zeit lang in dem Haushalt, oftmals zwei bis drei Monate und werden dann von einer anderen Kraft abgelöst. Wir sind hier also mit einer ausgeprägten Pendelmigration konfrontiert, sodass man die Zahl der aus Osteuropa stammenden Betreuungskräfte mindestens mit dem Faktor 2, eher mehr, multiplizieren muss. Dann bewegen wir uns in einer Größenordnung von 400.000 und darüber hinaus temporär in die Pflege und Betreuung ein- und wieder auspendelnden Personen.

Unabhängig von allen offenen arbeits- und sozialrechtlichen Fragen in diesem Bereich, bei denen bis heute seitens der politisch Verantwortlichen eine „Vogel-Strauß-Politik“ betrieben wird, ${ }^{15}$ kann und muss man plausibel davon ausgehen, dass die Zahl der aus Osteuropa nach Deutschland pendelnden Betreuungskräfte abnehmen wird - aufgrund der desaströsen demografischen Entwicklung in den Ländern dort, aber auch aufgrund des tendenziell abnehmenden Wohlstandsgefälles, das bislang der Hauptgrund dieser Form der temporären Zuwanderung war. Wenn das so sein sollte, dann wird das den Personalbedarf in der professionellen Altenpflege im Zusammenspiel mit

14 Zur Situation und kritischen Diskussion in Österreich, wo schon vor Jahren anders als in Deutschland den Versuch einer Teil-Legalisierung der „24-StundenPflege" unternommen wurde, vgl. den Beitrag von Aulenbacher et al. (2018), dort auch mit einem Blick auf die Rolle der Vermittlungsagenturen. Über einen Teil dieser Agenturen gibt es gerade wieder einmal eine sehr kritische Diskussion, vgl. hierzu beispielsweise die Reportage von Pramer et al. (2019).

15 Vgl. hierzu bereits Sell (2010). anderen Veränderungen auf Seiten des häuslichen Pflegepotenzials weiter erhöhen.

\subsection{Die Rekrutierung ausländischer Pflegekräfte oder: Vom Entstehen einer eigenen Branche; viele Klimmzüge, überschaubare Resultate}

Unstrittig ist, dass Krankenhäuser und Pflegeinrichtungen die Suche und Anwerbung ausländischer Pflegekräfte als einen Weg der Personalbeschaffung beschreiten (müssen) und sie tun das erwartungsgemäß mit unterschiedlicher Intensität, denn das ist sehr voraussetzungsvoll und auch mit teilweise erheblichen Kosten verbunden.

Eine Vorbemerkung: Wenn man sich nur auf die mittlerweile vorliegenden Veröffentlichungen stützt und diese nicht mit den realen Zahlen gewichtet, dann kann man den Eindruck gewinnen, dass wir es hier mit einer großen Zahl an Pflegekräften zu tun haben, die angeworben und ins Land geholt werden. Es wurde bereits aufgezeigt, dass das zu relativieren ist.

Auf der anderen Seite ist mittlerweile eine eigene Branche entstanden rund um das Thema Beschaffung ausländischer Pflegekräfte, was angesichts der vielfältigen und höchst speziellen Hürden, die man nehmen muss, nicht verwundert. Damit verbunden sind erhebliche Transaktionskosten, die von Seiten der Auftraggeber oftmals unterschätzt werden - und dass bei einer mehr als fragilen Angelegenheit, denn selbst wenn man Pflegekräfte aus dem Ausland gewinnen kann, bedeutet das noch lange nicht, dass sie auch auf Dauer bleiben (werden). ${ }^{16}$ Gerade die Erfahrungen der letz-

16 Rebekka Süss (2018) hat in ihrer Arbeit „Ökonomische Bewertung der Anwerbung ausländischer Pflegekräfte" für den Bereich der Universitätsklinika und Medizinischen Hochschulen eine genauere Kostenkalkulation der Rekrutierung ausländischer Pflegekräfte vorgenommen. 
ten Jahre mit den aus den südeuropäischen Krisenstaaten der EU angeworbenen Pflegekräften sollte zur Vorsicht mahnen. ${ }^{17}$

Chancen und Hemmnisse der internationalen Fachkräfterekrutierung ${ }^{18}$ waren beispielsweise Thema der Studie von Bonin et al. (2015) für die Bertelsmann-Stiftung. Die Unternehmen, für die eine Rekrutierung von Pflegefachkräften in Frage kommt, richten ihren Blick dabei fast durchweg auf die EU-Mitgliedsländer. Bei den Ländern außerhalb der Europäischen Union konzentrierten sich die Aktivitäten vor allem auf osteuropäische Staaten, während asiatische Entsendeländer wie China, die Philippinen und Vietnam eine sichtbare, aber kleine Rolle spielen.

Bonin et al. (2015, S. 64) kommen auf der Basis ihrer Befragung von Personalverantwortlichen, die Erfahrungen mit der Beschäftigung ausländischer Pflegekräfte gemacht haben, zu einem positiven Befund:

"Alles in allem haben die Unternehmen, die in den letzten drei Jahren Pflegefachkräfte aus dem Ausland eingestellt haben, mit diesen neuen Mitarbeitern positive Erfahrungen gemacht. Drei von fünf Personalverantwortlichen sind mit ihnen zufrieden oder sogar sehr zufrieden, nur einer von zehn ist unzufrieden oder sehr unzufrieden. Die Kompetenzen dieser Pflegefachkräfte werden überwiegend auf dem Niveau der aus dem Inland stammenden Kollegen

17 So sind viele der aus Spanien für die Krankenhauspflege rekrutierten Fachkräfte wieder zurückgegangen. Dafür gibt es natürlich viele und individuell sehr unterschiedliche Gründe, aber auffällig ist, wie oft von den Fachkräften aus anderen Ländern zum einen die reale Personalausstattung als unerträglich defizitär wahrgenommen wird, zum anderen wird immer wieder beklagt, wie „niedrig“ der Stellenwert des Pflegepersonals in Deutschland ist. Vgl. dazu beispielsweise bereits Kellner (2013).

18 Für eine generelle arbeitsmarktliche Einbettung und den vielfältigen Problemstellen vgl. die Arbeit von Mergener (2018): Zuwanderung in Zeiten von Fachkräfteengpässen auf dem deutschen Arbeitsmarkt. Einflussfaktoren auf die Beschäftigungs- und Rekrutierungschancen ausländischer Fachkräfte aus betrieblicher Perspektive. Speziell mit Blick auf die Rekrutierung von internationalen Pflegekräfte vgl. auch die Arbeit von Schreck (2017). gesehen. Lediglich im Hinblick auf die Praxiserfahrung sind die im Ausland rekrutierten Fachkräfte nach Einschätzung der Personalverantwortlichen klar im Nachteil. Dies dürfte damit zusammenhängen, dass die im Ausland vorwiegend akademisch organisierte Ausbildung für pflegerische Tätigkeiten deutlich weniger Praxisanteile umfasst als die Ausbildung von Pflegeschülern in Deutschland. Andererseits fällt das Urteil über die Einsatzbereitschaft der international rekrutierten Pflegefachkräfte sehr positiv aus. Die Hälfte der Personalverantwortlichen kommt zu der Einschätzung, dass ihr in jüngerer Zeit aus dem Ausland eingestelltes Pflegefachpersonal in dieser Hinsicht leistungsfähiger ist als ihre übrigen Pflegefachkräfte."

Bereits damals wurde seitens der Personalverantwortlichen auf den sehr hohen Aufwand hingewiesen. „Über die Hälfte berichten von einem hohen oder ziemlich hohen Aufwand, bis ihre in jüngster Zeit aus dem Ausland angeworbenen Pflegefachkräfte voll einsatzfähig waren. “ Hier interessant: „Ein Teil dieser Kosten kann zudem verloren sein, weil international rekrutierte Fachkräfte teilweise mit falschen Vorstellungen kommen und wieder in die Heimat zurückkehren oder nach kurzer Zeit das Unternehmen wechseln, was insbesondere im Bereich der Altenpflege vorkommt, aus der heraus international rekrutierte Pflegefachkräfte in die tendenziell besser bezahlte Gesundheitsund Krankenpflege wechseln."

Die Unternehmen, die in den letzten drei Jahren Nicht-EU-Bürger als Pflegefachkräfte rekrutiert haben, hatten zu zwei Dritteln Schwierigkeiten bei der Anerkennung ausländischer Qualifikationen und zu $60 \%$ Probleme beim Erlangen der Zuwanderungserlaubnisse. Und auch der angesprochene eigene Markt für Beratungs- und Vermittlungsdienstleister wurde kritisch angesprochen: „Tatsächlich gibt es beispielsweise Berichte über unzuverlässige und wenig leistungsfähige Personaldienstleister im Markt für internationales Recruiting" (Bonin et al. 2015, S. 67).

Mittlerweile ist mit Blick auf die Altenpflege realistische Skepsis eingekehrt. So schluss- 
folgert Stefan Etgeton von der BertelsmannStiftung: „Das Potenzial der Gewinnung und Integration von Pflegekräften aus dem Ausland sollte nicht überschätzt werden, zumal der Rekrutierungsaufwand erheblich ist" (Etgeton 2018, S. 2). Und Hackmann und Sulzer (2018) haben sich in ihrer Studie „Strategien gegen den Fachkräftemangel in der Altenpflege. Probleme und Herausforderungen“ ebenfalls relativierend zu Wort gemeldet: „Besonders ausländische Fachkräfte werden als mögliche Personalressource für die Altenpflege gesehen. Zahlreiche Projekte befassen sich mittlerweile mit dem Thema, wie beispielsweise das Welcome Center Sozialwirtschaft in BadenWürttemberg, oder das groß angelegte Projekt Triple Win ${ }^{19}$ der Zentralen Auslands- und Fachvermittlung (ZAV), der Bundesagentur für Arbeit und der Deutschen Gesellschaft für Internationale Zusammenarbeit (GIZ), das qualifizierte Pflegefachkräfte aus Serbien, BosnienHerzegowina und den Philippinen für die deutsche Altenpflege anwirbt" (Hackmann und Sulzer 2018, S. 41).

Aber: „Fraglich ist, inwiefern der Zugewinn an neuen Fachkräften dem Aufwand und den Kosten einer Rekrutierung im Ausland gerecht wird. In den Fachgesprächen wurde von der Mehrheit der Fachgesprächspartner/ -innen Maßnahmen zur Linderung des Personalmangels über eine Rekrutierung von ausländischen Fachkräften kritisch gesehen. Einerseits sind sprachliche und kulturelle Herausforderungen zu bedenken sowie die unterschiedlichen Studiums- und Ausbildungsinhalte bei ausländischen Berufsabschlüssen in den einzelnen Ländern. Andererseits wurde es kritisch gesehen, die Personalprobleme in Deutschland auf dem Rücken von anderen Ländern auszutragen, die möglicherweise selbst mit einem Personalmangel in der Pflege zu kämpfen haben“ (Hackmann und Sulzer 2018, S. 42).

Wenn man die Beispiele aus der Praxis studiert, wo es um den Einsatz ausländischer Pflegekräfte geht, dann stößt man immer wie-

19 Zum Projekt Triple Win vgl. die Website www.triplewin-pflegekraefte.de. der auf einen Aspekt, der von entscheidender Bedeutung ist und der von denen, die gerne die großen Lösungsansätze suchen, aber die Umsetzung dann nicht ausbaden müssen, vernachlässigt oder kleingeredet wird: die Sprachkenntnisse. Das zentrale Nadelöhr gerade für die Menschen, die in der Pflege arbeiten wollen oder sich das vorstellen können, ist oftmals die deutsche Sprache, denn man arbeitet in der Pflege nicht an Maschinen oder anderen Dingen, sondern am und mit dem Menschen, und da ist Kommunikation von zentraler Bedeutung. Und die deutsche Sprache ist keine einfache Sprache und nicht jeder ist sprachbegabt. Dann benötigt man Zeit und eine qualifizierte Begleitung der zukünftigen (potenziellen) Pflegekräfte, denn auf ausreichende Sprachkompetenz muss gerade in solchen Bereichen wie der Pflege besonderer Wert gelegt werden im beiderseitigen Interesse, sowohl der Pflegebedürftigen wie auch des Personals. Dazu auch die Befunde von Visel (2017). Sie diagnostiziert in ihrem Beitrag „Berufsanerkennung und , sichere Pflege - Die Bedeutung von Sprachkenntnissen in Anerkennungsverfahren für Pflege- und Gesundheitsberufe“, dass nach wie vor in der Praxis Hürden für die Antragsteller bestehen. Diese sind verbunden mit den geforderten deutschen Sprachkenntnissen. Im empirischen Teil wird gezeigt, wie vor allem Dimensionen von Sicherheit in Verbindung mit Sprache in Anerkennungsverfahren für Pflegekräfte relevant werden und weitere Hürden für die Antragsstellenden legitimieren.

Dazu ein Blick auf eine andere Profession: Die Sprachbarriere ist eine gewaltige und eben nur begrenzt aufhebbare Hürde. Hinzu kommen auch unverzichtbare fachliche Voraussetzungen, die man eben nicht beliebig absenken kann (was aber eine an sich zwingende Konsequenz wäre, wenn man dem Ziel einer Beschleunigung der und vor allem mehr Abschlussanerkennungen folgen würde). „Ärzte, die aus Drittstaaten stammen und in Deutschland ärztlich tätig sein wollen, müssen ihre medizinischen Kenntnisse bei den Kammern nachweisen. Und da hapert es gewal- 
tig“, so Anke Thomas in ihrem Artikel „Fast jeder zweite besteht Prüfung nicht“. Immer mehr Ärzte aus Syrien, Rumänien, Serbien, der Ukraine, Russland und Aserbaidschan wollen in Deutschland Fuß fassen und ärztlich tätig werden. Das sind die Länder, aus denen die größten Zuwachsraten zu verzeichnen sind. „Um zu zeigen, dass die Erhebung einer Anamnese, die Diagnosestellung oder das Schreiben eines Arztbriefes keine sprachlichen Probleme bereiten, müssen die Anwärter für die Tätigkeit in Deutschland eine Sprachprüfung absolvieren und außerdem auch ihre medizinischen Kenntnisse unter Beweis stellen. Laut einem aktuellen Bericht des MDR, der bei verschiedenen Ärztekammern nachgefragt hat, scheitern viele Ärzte an den Prüfungen. Die Durchfallquote bei den verpflichtenden Sprach- und Medizinprüfungen betrage im ersten Versuch mehr als $50 \%$. Allerdings können diese Versuche beliebig oft wiederholt werden. “ Und „natürlich“ erfolgen diese Prüfungen nicht etwa einheitlich, sondern sie sind Ländersache und variieren teilweise erheblich. Mit bedenklichen Streuungsbreiten - Beispiel Sprachtests bei Medizinern: „Während in Rheinland-Pfalz ein Sprachniveau von C1 geprüft werde, gäben sich andere Länder beziehungsweise Kammern teilweise noch mit einem niedrigeren Sprachniveau zufrieden“ (Thomas 2018). Schon C1 wäre kritisch zu diskutieren.

Und wenn wir eine ehrliche Diskussion führen würden, dann müsste man darauf hinweisen, dass wir tagtäglich unzählige Pflegeund vor allem Behandlungsfehler haben, weil es bei einem Teil der Beschäftigten enorme Sprachprobleme gibt. Aber man ist ja schon dankbar über jeden, der die lichten Reihen wieder auffüllt. ${ }^{20}$

20 Damit hier keine Missverständnisse aufkommen bereits heute sind die ausländischen Pflegekräfte und Ärzte (vor allem aus Osteuropa) eine Stütze in den Systemen, ohne die diese erhebliche Probleme bekommen würden. Mehr als 30.000 ausländische Ärzte sind hier bei uns berufstätig, in vielen Krankenhäusern würde der Normalbetrieb zusammenbrechen, wenn die Ärztinnen und Ärzte aus Rumänien
Die strukturell bedingte Hilflosigkeit wird auch an dem bereits bekannten, von vielen interessierten Kreisen gerne zitierten Textbaustein mit der schnelleren Anerkennung der ausländischen Abschlüsse sowohl in Pflege wie auch bei den Ärzten erkennbar. Hört sich vernünftig an, verspricht aber mehr, als es halten kann. Denn das strukturelle Dilemma, das hier zu benennen ist, bezieht sich auf einen Aspekt, der jenseits der formalen Gleichwertigkeit von Abschlüssen liegt. Und das ist nicht nur die Sprache, wie beschrieben, sondern gerade in der Altenpflege sind es auch geteilte Erfahrungen und Mentalitäten, die man schlichtweg nicht haben kann, wenn man aus fernen Ländern nach Deutschland importiert wird. Und das kulturelle Verständnis ist eine eigene, wichtige Dimension guter Pflege, gerade in der Altenpflege.

Aber die politische Maschinerie ist zwischenzeitlich in Gang gesetzt worden. Beispielsweise mit der im vergangenen Jahr ins Leben gerufenen „Konzertierten Aktion Pflege" der drei Ministerien für Gesundheit, Arbeit und Familien. Bislang dringt noch nicht wirklich etwas nach draußen (und wenn, dann wird es vorläufig dementiert, wie das folgende Beispiel). Was wir wissen: es wurden fünf Arbeitsgruppen eingerichtet:

Arbeitsgruppe 1 - "Ausbildung und Qualifizierung"

Arbeitsgruppe 2 - „Personalmanagement, Arbeitsschutz und Gesundheitsförderung"

Arbeitsgruppe 3 - „Innovative Versorgungsansätze und Digitalisierung"

Arbeitsgruppe 4 - „Pflegekräfte aus dem Ausland"

Arbeitsgruppe 5 - „Entlohnungsbedingungen in der Pflege"

Auch mit dabei: der Arbeitgeberverband Pflege (AGVP) und der bpa Arbeitgeberverband. Und von dieser Seite wird eine Menge Hoffnung in

und Bulgarien ihre Koffer packen und in die Länder zurückgehen würden, die sie übrigens ausgebildet haben und die unter erheblichen Versorgungsproblemen leiden, weil ihre Fachkräfte angesichts der Wohlstandskluft individuell auch verständlich im Ausland ihr Glück suchen, aber in den Heimatländern Riesen-Lücken gerissen haben. 
den Ansatz einer Absenkung der Fachkraftquote mit dem Ziel, mehr un- und angelerntes (und damit natürlich auch kostengünstigeres) Personal beschäftigen zu können, sowie in die Personalbeschaffung im Ausland gesetzt. Bereits im August 2018 wurde dann über eines der geplanten Pilotprojekte berichtet (und kurz darauf wurde seitens des Bundesgesundheitsministeriums dementiert, dass es so etwas schon geben würde):

„Unter Koordination der Ministerien für Gesundheit und Arbeit wurde ein zwei Jahre laufendes Projekt gestartet, mit dem bis zu 15.000 ausländische Pflegefachkräfte für die Versorgung in Deutschland gewonnen werden sollen. Dazu soll auf Bundesebene eine zentrale Anerkennungsstelle eingerichtet werden. Ferner wird es eine Koordinierungsstelle geben, die Rekrutierungsagenturen zertifiziert, für die schnelle Visa-Erteilung sorgt und die Sprachförderung für die ausländischen Pflegekräfte organisiert. Auf Initiative des Arbeitgeberverbandes Pflege wurde bereits am 17. Mai die Bundesarbeitsgemeinschaft Ausländische Pflegekräfte gegründet. Sie ist eine landesweite trägerübergreifende Plattform für einen Erfahrungsaustausch und die Vernetzung aller an der Rekrutierung und Beschäftigung ausländischer Pflegekräfte beteiligten Organisationen. Der Arbeitgeberverband Pflege fordert nun, dass Ausländer, die innerhalb von sechs Monaten als Fachkraft anerkannt werden und einen Arbeitsvertrag haben, in Deutschland ein Bleiberecht erhalten“ (Sell 2018b).

In der Arbeitsgruppe 4 der „Konzertierten Aktion Pflege“, wo man sich speziell mit dem Thema Pflegekräfte aus dem Ausland befasst, fokussiert man derzeit offensichtlich auf einen „Beschleunigungsansatz“ hinsichtlich der Verwaltungsverfahren und des Handelns im Inund Ausland. Auch und gerade die Zuständigkeitszersplitterungen in den Bundesländern sind Thema der Arbeitsgruppe. Man wird erwarten können, dass es Handlungsempfehlungen geben wird, die auf eine deutliche Zentralisierung der Anerkennungsverfahren hin- auslaufen werden. ${ }^{21}$ Dann wird sich natürlich die Frage stellen, ob und in welchem Ausmaß die Bundesländer bereit sein werden, die ihnen zugewiesenen Aufgaben an eine bundesweite Organisation zu delegieren.

Die Vorstellung, das Ausland sei irgendwie ein Steinbruch, an dem man sich bedienen kann, wird auch von dem sozialdemokratischen Bundesarbeitsminister Hubertus Heil geteilt, der zudem auch noch aus nationaler Sicht durchaus konsequent für eine Strategie der Rosinenpickerei plädiert: Heil will befristete Visa unter anderem für Pflegekräfte. Man sollte sich das aufmerksam zur Kenntnis nehmen, was er sagt: In der Debatte um das geplante Fachkräfte-Einwanderungsgesetz ist Bundesarbeitsminister Hubertus Heil (SPD) dafür, Bewerber aus besonders nachgefragten Berufen wie der Pflege eine befristete Einreise zum Zweck der Arbeitssuche zu gestatten. „Ich kann mir vorstellen, dass Pflegekräfte aus dem Ausland für ein halbes Jahr nach Deutschland kommen und sich hier Arbeit suchen" ... Sollte ihnen das nicht gelingen, müssten sie nach $\mathrm{Ab}$ lauf der Zeit wieder zurück: „Der Bezug von Sozialleistungen muss natürlich ausgeschlossen sein.“ Bei dem Gesetz dürfe es „nicht nur um höchstqualifizierte Arbeitnehmer" gehen, betonte Heil. Es sei wichtig, „möglichst unbürokratische Prozesse einzurichten“.

21 Dazu Szepan (2019): „Eine Option wäre es, die zentrale Gutachtenstelle für Gesundheitsberufe (GfG) auszubauen und die Verfahren und Kompetenzen zu bündeln. Idealerweise würde die GfG den Antrag auf Gleichwertigkeit entgegennehmen, die Gleichwertigkeitsprüfung durchführen und nach Prüfung der Echtheit der Unterlagen rechtsverbindlich den Bescheid erteilen. Gegenstand der Prüfung wäre auch die detaillierte inhaltliche Begutachtung der Ausbildung anhand der eingereichten Unterlagen und eine Bewertung der Berufserfahrung, durch die beispielsweise Unterschiede in der Ausbildung ausgeglichen werden könnten. Damit würde dieses Vorgehen mit der im Entwurf des Fachkräfte-Einwanderungsgesetzes vorgesehenen zentralen Ausländerbehörde in Einklang stehen." 
Das muss man erst einmal sacken lassen. Mittlerweile ist das in den offiziellen Entwurf eines Fachkräfte-Einwanderungsgesetzes eingeflossen. Dort heißt es: „Die Möglichkeiten des Aufenthalts zur Arbeitsplatzsuche für Fachkräfte werden in einer Norm zusammengefasst. Für Fachkräfte mit Berufsausbildung wird die Möglichkeit zur befristeten Einreise zur Arbeitsplatzsuche analog zur Regelung für Fachkräfte mit akademischer Ausbildung geschaffen und für fünf Jahre befristet erprobt. Zudem wird der Aufenthalt zu ergänzenden Qualifizierungsmaßnahmen für Drittstaatsangehörige mit im Ausland abgeschlossener Berufsbildung im Rahmen der Anerkennung ausländischer Berufsqualifikationen erweitert und attraktiver gestaltet und unter Einbindung der Bundesagentur für Arbeit eine begrenzte Möglichkeit geschaffen, unter bestimmten Voraussetzungen die Anerkennung erst in Deutschland durchzuführen."

Eine generell kritische Auseinandersetzung mit diesem Ansatz findet man bei Seils (2018) - allerdings nicht mit Blick auf die Pflege. So schreibt er in seinem Fazit: „Sieht man also von den kurzfristigen Gewinnen der Unternehmen ab, dann spricht wenig für die Einwanderung zur Arbeitssuche. Sie ist weder erforderlich noch sinnvoll. Sollte sie dennoch umgesetzt werden, ist statt des möglichen Ausschlusses einiger Berufsgruppen eine Beschränkung der Einwanderung zur Arbeitssuche auf ausgewählte Mangelberufe, etwa in Pflege- und Gesundheitsberufen, sinnvoll. Hier wäre die Konkurrenz mit den einheimischen Arbeitskräften begrenzt und die Wahrscheinlichkeit, dass es den Einwanderern gelingt, in den sechs Monaten eine Anstellung zu finden, vergleichsweise hoch."

Da ist sie wieder, die Hoffnung, dass es in der Pflege funktionieren wird. Aber bei allem Verständnis für Hoffnungen bleibt zum jetzigen Zeitpunkt nur, eine Menge Wasser in den aufgetischten Wein zu gießen, sowohl hinsichtlich der quantitativen wie auch besonders mit Blick auf die kaum diskutierten qualitativen Dimensionen.
Mit diesen beiden Dimensionen beschäftigt sich auch die Studie von Pütz et al. (2019). Dort hat man sich hinsichtlich der quantitativen Größenordnung die Anerkennungsstatistik angeschaut, bei der es um die im In- und Ausland gestellten Anträge auf Anerkennung der Gleichwertigkeit von Berufsabschlüssen geht (vgl. zu dieser Datenquelle auch die differenzierte Darstellung bei Schmitz und Winnige 2019). Nach den Angaben in Pütz et al. (2019) ist die Zahl der Fachkräfte für Gesundheitsund Krankenpflege, die jährlich aus dem Ausland nach Deutschland kommen, zuletzt auf fast das Sechsfache gestiegen: ${ }^{22}$ Von knapp 1.500 im Jahr 2012 auf 8.800 im Jahr 2017. Größtenteils stammen sie aus ost- und südeuropäischen Staaten außerhalb der EU oder von den Philippinen. Zwar ist die Bundesrepublik laut der neuen Untersuchung im internationalen Vergleich noch weit davon entfernt, als etabliertes Zielland der globalisierten Pflegefachkräftemigration zu gelten: 2010 hatten knapp $6 \%$ der Pflegerinnen und Pfleger ihre Ausbildung im Ausland absolviert. In Großbritannien oder der Schweiz lag der Anteil zwei- bis dreimal so hoch. Aber die Zunahme in den vergangenen Jahren ist offensichtlich. Dabei kommen die meisten neuen Pflegekräfte bislang aus der europäischen Nachbarschaft: Rumänien, Kroatien, Polen und Ungarn stellten 2017 die wichtigsten EU-Herkunftsländer; hinzu kamen Bosnien-Herzegowina, Serbien und Albanien als die benachbarten Drittstaaten. Oft sind die Neueinstellungen Ergebnis gezielter Anwerbeaktionen. In den Herkunftsländern haben sich professionelle Agenturen auf die Vermittlung von qualifiziertem Gesundheitspersonal spezialisiert, das mit Sprachkursen auf die Arbeit in Deutschland vorbereitet wird.

Der Fokus der Studie liegt auf der Frage nach der betrieblichen Integration der zugewanderten Pflegekräfte. Sowohl neu migrierte

22 Für eine Zusammenfassung vgl. diesen Beitrag: Pflegefachkräfte aus dem Ausland: Zahl hat sich versechsfacht - nicht selten Konflikte wegen Unterschiede in Ausbildung und Berufsverständnis, https://www.boeckler.de/117819_118702.htm. 
als auch einheimische Beschäftigte - von denen selbst etliche einen Migrationshintergrund haben - sind oft unzufrieden mit der Zusammenarbeit. Differenzen und Missverständnisse, die häufig auf Unterschieden in der Ausbildung und der gewohnten Arbeitsteilung zwischen medizinischem Personal, Pflege- und Hilfskräften beruhen, werden nicht selten stereotyp mit „kulturellen Unterschieden“ erklärt. Das kann Konflikte ebenso verschärfen wie die generell oft schwierigen Arbeitsbedingungen, die ja eigentlich verbessert werden sollen.

Die hier angedeuteten Konflikte werden auf der Basis einer qualitativen Erhebung über „knapp 60 ausführliche Interviews“ (speziell zum Bereich der Krankenhauspflege dazu auch ergänzend die Studie von Rand und Larsen 2019) so beschrieben: Offensichtlich gibt es auf beiden Seiten erhebliche Differenzen bei Ausbildung, beruflichem Selbstverständnis und gewohnter Arbeitsorganisation: In vielen der Herkunftsländer werden Pflegefachkräfte an Hochschulen ausgebildet. Eine hochqualifizierte schulisch-betriebliche Ausbildung wie in Deutschland ist dort unbekannt. Gleichzeitig übernehmen Pflegefachkräfte etwa in Südeuropa in der Tendenz mehr Management- sowie Behandlungsaufgaben, die in Deutschland Medizinern vorbehalten sind. Tätigkeiten der so genannten „Grundpflege“ auszuüben, also etwa Patientinnen und Patienten beim Essen oder der Körperpflege zu unterstützen, ist dort für Pflegefachkräfte ungewöhnlich. Dafür gibt es, mehr noch als in Deutschland, teils spezielle Service-Kräfte, teilweise müssen Angehörige einspringen. Daraus resultieren im Arbeitsalltag zahlreiche Spannungen: So haben viele der befragten zugewanderten Pflegekräfte das Gefühl, „unter Wert“ arbeiten zu müssen, sie fühlen sich häufiger von Informationen ausgeschlossen, von Vorgesetzten schlechter behandelt.

Die in Deutschland ausgebildeten Pflegefachkräfte kritisieren wiederum, dass neu zugewanderte Kolleginnen und Kollegen schon wegen mangelnder Sprachkenntnisse im verantwortungsvollen und eng getakteten, stres- sigen Arbeitsalltag nicht voll einsetzbar seien. Die akademische Ausbildung im Ausland wird oft nicht als Vorteil gesehen, sondern als „praxisfern" kritisiert. Dafür fehlten grundsätzliche Kompetenzen, etwa bei der Körperpflege von Patienten und im „Sozialverhalten“. Aus der Sicht der befragten einheimischen Beschäftigten können die Fachkräfte aus dem Ausland daher zumindest für einen längeren Einarbeitungszeitraum allenfalls als „Schüler“ beschäftigt werden. Das kann dazu führen, dass ein bereits vorhandener Mangel an Pflegepersonal durch die neuen Kräfte nicht gemildert, sondern in der Wahrnehmung und Realität der „einheimischen“ Pflegefachkräfte sogar noch verschärft werden kann.

Fazit: Die seit langem bekannte und immer wieder reanimierte Hoffnung, über das Ausland unsere Personalprobleme lösen zu können, wird genau so funktionieren wie in den zurückliegenden Jahrzehnten. Also flächendeckend und in umfassender Art und Weise nicht. Allenfalls eine punktuelle Entlastung wird es geben können für das eine oder andere Krankenhaus oder das eine oder andere Pflegeheim.

\section{Literatur}

Afentakis A, Maier T (2014) Können Pflegekräfte aus dem Ausland den wachsenden Pflegebedarf decken? Analysen zur Arbeitsmigration in Pflegeberufen im Jahr 2010. Wirtsch Stat 3:173-180

Aulenbacher B, Leiblfinger M, Prieler V (2018) Ein neuer Sorgemarkt im Wohlfahrtsstaat: 24-StundenBetreuung in Österreich und Dienstleistungsangebote von Wiener Vermittlungsagenturen. In: Filipič U, Schönauer A (Hrsg) Zur Zukunft von Arbeit und Wohlfahrtsstaat. Perspektiven aus der Sozialforschung. ÖGB Verlag, Wien, S 47-56

Bonin H, Braeseke G, Ganserer A (2015) Internationale Fachkräfterekrutierung in der deutschen Pflegebranche. Chancen und Hemmnisse aus Sicht der Einrichtungen. Bertelsmann Stiftung, Gütersloh

Bundesagentur für Arbeit (2019) Beschäftigte nach Berufen (KIdB 2010; Quartalszahlen). Stichtag: 30. Juni 2018, Nürnberg, 10. Januar 2019

Deutscher Paritätischer Wohlfahrtsverband (2018) Handreichung Anwerbung von Pflegekräften aus dem 
Ausland für den deutschen Arbeitsmarkt. Deutscher Paritätischer Wohlfahrtsverband, Berlin

Etgeton S (2018) Themenpapier Strategien gegen den Fachkräftemangel in der Altenpflege. Bertelsmann Stiftung, Gütersloh

Flake R et al (2018) Fachkräfteengpass in der Altenpflege - Status quo und Perspektiven. IW-Trends 3:21-39

Hackmann T, Sulzer L (2018) Strategien gegen den Fachkräftemangel in der Altenpflege. Probleme und Herausforderungen. Prognos, Basel

Hielscher V, Kirchen-Peters S, Nock L (2017) Pflege in den eigenen vier Wänden: Zeitaufwand und Kosten. Pflegebedürftige und ihre Angehörigen geben Auskunft. Study Bd. 363. Hans-Böckler-Stiftung, Düsseldorf

Kellner HG (2013) „Krankenpfleger sind in Deutschland Hilfskräfte". Deutschlandfunk Online, 07.10.2013. https://www.deutschlandfunk.de/erfahrungeneiner-spanierin-krankenpfleger-sind-in.795.de. html?dram:article_id=264297\&xtor=AD-251-\%5B \%5D-\%5B\%5D-\%5B\%5D-\%5Bdlf-mobil\%5D-\%5B \%5D-\%5B\%5D. Zugegriffen: 9. Jan. 2019

von der Malsburg A, Isfort M (2014) Haushaltsnahe Dienstleistungen durch Migrantinnen in Familien mit Pflegebedürftigkeit. 24 Stunden verfügbar - Private Pflege in Deutschland. Friedrich-Ebert-Stiftung, Bonn

Mergener A (2018) Zuwanderung in Zeiten von Fachkräfteengpässen auf dem deutschen Arbeitsmarkt. Einflussfaktoren auf die Beschäftigungs- und Rekrutierungschancen ausländischer Fachkräfte aus betrieblicher Perspektive. Bundesinstitut für Berufsbildung, Bonn

Nürnberg V, Traoré S (2019) Können Arbeitsmigranten den Fachkräftemangel beseitigen? Zuwanderung und Pflegenotstand im Krankenhaus. Krankenhaus 2:124-126

Pramer P et al (2019) Als man Elena die Stimme nahm. Eine 24-Stunden-Betreuerin prangert die Missstände in ihrer Branche an und zieht gegen die ausbeuterischen Methoden der Vermittlungsagenturen ins Feld. Diese wollen sie mit allen Mitteln zum Schweigen bringen. Der Standard Online, 20. Jan. 2019. https://derstandard.at/2000096455290/ Misstaende-bei-24-Stunden-Pflege-Als-manElena-die-Stimme. Zugegriffen: 9. Jan. 2019

Pütz R et al (2019) Betriebliche Integration von Pflegefachkräften aus dem Ausland. Innenansichten zu Herausforderungen globalisierter Arbeitsmärkte. Studie der Hans-Böckler-Stiftung, Nr. 416. HansBöckler-Stiftung, Düsseldorf

Rand S, Larsen C (2019) Herausforderungen und Gestaltung betrieblicher Integration von Pflegefachkräften. Einblicke aus der Krankenhauspraxis. Working Paper Forschungsförderung, Nr. 114. Hans-BöcklerStiftung, Düsseldorf
Schmitz N, Winnige S (2019) Anerkennung ausländischer Berufsqualifikationen: Anträge aus dem Ausland im Spiegel der amtlichen Statistik. Ergebnisse des BIBBAnerkennungsmonitorings. Bundesinstitut für Berufsbildung (BIBB), Bonn (Vorabversion Januar 2019)

Schreck C (2017) Rekrutierung von internationalen Pflegefachkräften. Chancen und Herausforderungen für den Fachkräftemangel in Deutschland. Springer, Wiesbaden

Seils E (2018) Einwanderung zur Arbeitsuche? Policy Brief WSI Nr. 27. Wirtschafts- und Sozialwissenschaftliches Institut, Düsseldorf

Sell S (2010) Abschied von einer "Lebenslüge" der deutschen Pflegepolitik. Plädoyer für eine "personenbezogene Sonderregelung" und für eine aktive Gestaltung der Beschäftigung von ausländischen Betreuungs- und Pflegekräften in Privathaushalten. Remagener Beiträge zur Sozialpolitik 09-2010. Hochschule Koblenz, Remagen

Sell S (2018a) Die einen wollen Tariflöhne in der Altenpflege, die anderen die Arbeitgeber genau davor bewahren. Der Weg wird kein einfacher sein. Aktuelle Sozialpolitik, 01.07.2018. http://aktuellesozialpolitik.de/2018/07/01/die-einen-wollentarifloehne-in-der-altenpflege-die-anderen-diearbeitgeber-davor-bewahren/. Zugegriffen: 9. Jan. 2019

Sell S (2018b) Konzertierte Aktion Arbeitgeber-Pflege? Aktuelle Sozialpolitik, 14.08.2018. http://aktuellesozialpolitik.de/2018/08/14/konzertierte-aktionarbeitgeber-pflege/. Zugegriffen: 9. Jan. 2019

Sell S (2019) Wie viele Pflegekräfte in der Altenpflege müssen es denn sein? Von (un)sicheren Bedarfen und beweglichen Zielen bei der Diskussion über den bestehenden und kommenden Mangel an Pflegepersonal. Remagener Beiträge zur Sozialpolitik 232019. Hochschule Koblenz, Remagen

Siyam A et al (2013) Monitoring the implementation of the WHO global code of practice on the international recruitment of health personnel. Bull World Health Organ 91:816-823

Süss R (2018) Ökonomische Bewertung der Anwerbung ausländischer Pflegekräfte. Eine Studie an deutschen Universitätskliniken und Medizinischen Hochschulen. Springer Gabler, Wiesbaden

Szepan NM (2019) Mehr Köpfe für die Pflege. Gesundheit und Gesellschaft digital 02/2019. https://www. gg-digital.de/2019/02/thema-des-monats/mehrkoepfe-fuer-die-pflege/index.html. Zugegriffen: 9. Jan. 2019

Tankwanchi A et al (2014) Has the WHO global code of practice on the international recruitment of health personnel been effective? Lancet 2:e390-e391

Thomas A (2018) Fast jeder zweite besteht Prüfung nicht. Ärzte Zeitung Online, 26.03.2018. https://www.aerztezeitung.de/politik_gesellschaft/ 
berufspolitik/article/960501/zulassung-fast-jederzweite-auslaendische-arzt-besteht-pruefung-nicht. html. Zugegriffen: 9. Jan. 2019
Visel S (2017) Berufsanerkennung und Bichere Pflege"Die Bedeutung von Sprachkenntnissen in Anerkennungsverfahren für Pflege- und Gesundheitsberufe. Sozialer Fortschr 66(10):635-650

Open Access Dieses Kapitel wird unter der Creative Commons Namensnennung 4.0 International Lizenz (http:// creativecommons.org/licenses/by/4.0/deed.de) veröffentlicht, welche die Nutzung, Vervielfältigung, Bearbeitung, Verbreitung und Wiedergabe in jeglichem Medium und Format erlaubt, sofern Sie den/die ursprünglichen Autor(en) und die Quelle ordnungsgemäß nennen, einen Link zur Creative Commons Lizenz beifügen und angeben, ob Änderungen vorgenommen wurden.

Die in diesem Kapitel enthaltenen Bilder und sonstiges Drittmaterial unterliegen ebenfalls der genannten Creative Commons Lizenz, sofern sich aus der Abbildungslegende nichts anderes ergibt. Sofern das betreffende Material nicht unter der genannten Creative Commons Lizenz steht und die betreffende Handlung nicht nach gesetzlichen Vorschriften erlaubt ist, ist für die oben aufgeführten Weiterverwendungen des Materials die Einwilligung des jeweiligen Rechteinhabers einzuholen. 